\title{
VARICOCELE: A PRINCIPAL CAUSA DA INFERTILIDADE MASCULINA
}

\section{Renata Pâmella Fonseca}

Biomédica; Acadêmica do curso de Farmácia do Centro Universitário de Maringá (UNICESUMAR), Maringá (PR), Brasil.

\section{Luciana Conci Macedo}

Bióloga; Doutora em Biociências e Fisiopatologia; Pós Doutoranda em Biociências e Fisiopatologia pela Universidade Estadual de Maringá (UEM); Docente da Faculdade Ingá, Maringá (PR), Brasil.

\begin{abstract}
RESUMO: Na população de homens saudáveis, a incidência geral de varicocele é de $10 \%$ a $15 \%$. Entre homens que procuram um serviço de reprodução assistida essa taxa sobe para 38\%. A varicocele é uma condição que envolve a dilatação do plexo pampiniforme, que drena o sangue dos testículos. O fluxo sanguíneo reverso é impedido por pequenas válvulas de sentido único. Defeitos nestas válvulas ou de compressão podem provocar dilatação do vaso e diminuição do volume testicular. $\mathrm{O}$ exame físico é fundamental para o seu diagnóstico e deve ser realizado em ambiente de temperatura não refrigerada, o que favorece o relaxamento da musculatura escrotal. A varicocele pode ser corrigida através do tratamento cirúrgico ou por embolização percutânea. A reprodução assistida demonstrou taxas de sucesso em relação à concepção, com o emprego de gametas obtidos diretamente dos testículos. A detecção precoce dessa alteração e seu tratamento cirúrgico eliminam com sucesso mais de $90 \%$ das varicoceles. Desta forma a correção cirúrgica permanece uma ótima opção para devolver a fertilidade ao casal com uma relação custo-benefício muito superior a qualquer procedimento de reprodução assistida. Assim, o objetivo desse trabalho é verificar, através de uma revisão bibliográfica, a prevalência de homens com infertilidade causada pela varicocele, processos fisiopatológicos, diagnóstico, suas formas de tratamento e a importância do auxílio das técnicas de reprodução assistida nos pacientes afetados.
\end{abstract}

PALAVRAS-CHAVE: Reprodução Assistida; Varicocele; Infertilidade;

\section{VARICOCELE: THE MAIN CAUSE FOR MALE INFERTILITY}

\begin{abstract}
General occurrence of varicocele in healthy male population lies between 10 and $15 \%$ and percentage rises to $38 \%$ among males who seek assisted reproduction service. Varicocele is a condition that involves the swelling of the pampiniform plexus that drains blood from the testicles. Reverse blood flow is impaired by small contrary valves, while defects in the valves or compression may cause the swelling of the vessel and a decrease of the testicle volume. A physical examination is basic for diagnosis and should be undertaken in a non-refrigerated environment which favors relax of the scrotal muscle. Varicocele may be corrected by surgery or by percutaneous embolization. Assisted reproduction has produced success rates with regard to conception through the use of gametes obtained directly from the testicles. Early detection of alterations and surgery eliminate successfully more than $90 \%$ of varicocele. Surgery is still the best option for fertility with higher cost-benefit than any other assisted reproduction. Current paper is a bibliographical review to verify the prevalence of males suffering from infertility caused by varicocele, physiopathological processes, diagnostic treatments and importance of reproduction techniques in patients.
\end{abstract}

KEY WORDS: Assisted Reproduction; Varicocele; Infertility. 


\section{INTRODUÇÃO}

A Organização Mundial da Saúde (OMS) estima que aproximadamente 80 milhões de pessoas ao redor do mundo apresentam problemas relacionados a infertilidade. De acordo com a Sociedade Americana de Medicina Reprodutiva (ASRM), a infertilidade é considerada uma doença do sistema reprodutor, cujas funções não se encontram em estado normal (COCUZZA, 2011).

A infertilidade é definida após um ano de coito desprotegido com atividade sexual regular e sem concepção (SOLIGO et al., 2007). As doenças do trato genital feminino são responsáveis por $50 \%$ a $60 \%$ dos casos, ao passo que $40 \%$ a $50 \%$ estão relacionados a fatores masculinos (BORGES et al., 2005).

A infertilidade masculina possui várias causas como obstrução, criptorquidia, infecções secundárias, fatores imunológicos, hormonais e genéticos. Se uma baixa contagem de espermatozóides é devida a um desequilíbrio hormonal, a administração dos hormônios apropriados pode aumentar a produção de espermatozóides (LEWIS, 2004). O normal é ter 20 milhões de espermatozóides por mililitro de esperma e que 50\% deles sejam móveis, isto é, tenham a capacidade de sair da vagina e chegar à trompa para encontrar o óvulo (GLINA, 2010).

Em alguns casos o sistema imunológico de um homem produz anticorpos IgA que envolvem o espermatozóide e o impede de fecundar o ovócito. Os fatores genéticos atuam na codificação de receptores de andrógenos ou hormônios de fertilidade, ou que regulam a motilidade e desenvolvimento de espermatozóides. Em geral a maioria das causas de infertilidade masculina é genética (LEWIS, 2004). A infertilidade é um fenômeno universal que afeta de $10 \%$ a $15 \%$ dos casais em idade reprodutiva (SOLIGO et al., 2007).

Também contribuem para a infertilidade, as causas de infecções secundárias, as doenças sexualmente transmissíveis, como prostatites, orquiepididimites e uretrites, que apresentam como agentes causais a Chlamydia trachomatia, Ureaplasma urealyticum e Mycoplasma, além dos agentes gram - negativos, como Escherichia coli, Pseudomonas aeruginosa e
Enterobacter (SAADE, 2005).

$\mathrm{Na}$ população em geral, a incidência de varicocele clínica é relatada entre $10 \%$ e 15\%, no entanto, em pacientes com incidência de infertilidade primária é entre $30 \%$ a $50 \%$, em pacientes com infertilidade secundária em até $80 \%$, razão pela qual é postulado que os efeitos da varicocele na fertilidade possuem uma deterioração progressiva (VASQUEZ et al., 2009).

A varicocele é considerada a principal causa de infertilidade masculina. É uma condição que envolve a dilatação do plexo pampiniforme, que drena o sangue dos testículos. Normalmente, o fluxo sanguíneo reverso é impedido por pequenas válvulas de sentido único. Defeitos nestas válvulas ou de compressão das veias por estruturas adjacentes podem provocar dilatação do vaso e diminuição do volume testicular (COCUZZA et al., 2008).

A varicocele é geralmente uma doença assintomática. É por isso que a maioria dos casos é diagnosticada em exames físicos de rotina como achados acidentais. No entanto, a varicocele pode apresentar sintomas como dor testicular e sensação de peso na região escrotal. O sinal mais comum é a diminuição do volume testicular, que pode chegar à atrofia. A maioria dos homens com varicocele pode nunca saber de sua existência (VASQUEZ et al., 2009).

Apesar das excelentes taxas de sucesso com o tratamento cirúrgico da varicocele, muitos profissionais ainda questionam a validade da realização da varicocelectomia e indicam tratamento com reprodução assistida (PASQUALOTTO et al., 2007).

A reprodução assistida envolve um conjunto de técnicas como relação programada, fertilização in vitro clássica e a fertilização in vitro por meio de injeção intracitoplasmática de espermatozóide (ICSI), que possui uma equipe multidisciplinar que participa no acompanhamento e desenvolvimento das técnicas (FREITAS; SIQUEIRA; SEGRE, 2008).

Essa maior incidência de varicocele em pacientes que procuram assistência médica talvez reflita apenas a ideia geral dos médicos que os encaminham para um especialista em infertilidade masculina ou até clínicas de reprodução humana, que acreditam ser a varicocele a grande causadora de infertilidade masculina (PASQUALOTTO et al., 2007). 
A relevância da reparação da varicocele através das tecnologias de reprodução assistida ainda está sujeita a debate por causa da divergência em resultados de pesquisas (BAAZEEM et al., 2011; NEVOUX et al., 2011). A escolha da técnica para reparo da varicocele e o impacto do processo sobre os parâmetros de qualidade do sêmen são também muito debatidos. A técnica de tratamento ideal seria estar associada com mais baixas taxas de recorrência e de complicações possíveis (PRASIVORAVONG et al., 2014).

Menkeveld et al. demonstraram que a medição ou avaliação da morfologia espermática permanece, portanto, uma ferramenta muito importante no diagnóstico do potencial de fertilidade de um homem e na decisão clínica tomada para o tratamento de pacientes com problemas de infertilidade. (MENKVELD et al.)

Este estudo tem como objetivo verificar através de uma revisão bibliográfica a prevalência de homens com infertilidade causada pela varicocele, processos fisiopatológicos, diagnóstico, suas formas de tratamento e a importância do auxílio das técnicas de reprodução assistida nos pacientes afetados.

\section{METODOLOGIA}

Este trabalho trata-se de uma revisão bibliográfica sobre a varicocele que foi realizada a partir de coletas em livros, revistas e artigos científicos obtidos nos últimos 20 anos, a partir de banco de dados como Bireme, Medline, PubMed, ScienceDirect e Scielo. Utilizaramse os seguintes descritores: varicocele, infertilidade, varicocelectomia e reprodução assistida. Após a coleta e leitura dos materiais, as informações foram selecionadas, discutidas, interpretadas e descritas no texto.

\section{FISIOPATOLOGIA}

Aproximadamente 85\% dos casais que tentam ter filhos atingem seu desejo, no máximo, em um ano. Os que não conseguem conceber nesse período, cerca de $40 \%$, são em consequência de problemas que advêm do homem ou tem auxílio dele (ABDELMASSIH, 2004).
Muitos fatores podem causar a infertilidade masculina. A varicocele é considerada a principal causa de infertilidade entre os homens. É uma condição que envolve a dilatação do plexo pampiniforme, que drena o sangue dos testículos. Normalmente, o fluxo sanguíneo reverso é impedido por pequenas válvulas de sentido único. Defeitos nestas válvulas ou de compressão das veias por estruturas adjacentes podem provocar dilatação do vaso e diminuição do volume testicular (COCUZZA et al., 2008).

Várias teorias foram propostas para explicar a ação da varicocele nos testículos. Entre elas estão a da temperatura, pela qual o refluxo sanguíneo provocaria um aumento, causando danos à produção espermática, e a do refluxo de epinifrina e norepinifrina, que traria da veia renal substâncias de ação adrenérgica, que atuariam ao nível das arteríolas testiculares, causando vasoconstrição, diminuição de oxigênio e aumento de gás carbônico (SCHEFFER et al., 2003), provocando hipóxia testicular devido à estase venosa que afeta a pressão parcial de oxigênio e altera o metabolismo aeróbio no testículo (PADUCH; SKOOG, 2001).

Vários outros fatores têm sido postulados, como oclusão de pequenos vasos, levando à disfunção nas células germinativas e de Leydig, diminuição na secreção de andrógenos, e insuficiente remoção ou retorno de substâncias tóxicas de origens renal e adrenal (PASQUALOTTO et al., 2007).

A ação da varicocele se desenvolveria em duas etapas: a primeira, sobre as células de Sertoli, causando danos sobre o epitélio germinativo e as células apresentariam vacúolos e se tornariam delgadas, não suportando a espermatogênese e deslizariam para a luz tubular, o que alteraria a formação das espermátides e, consequentemente, a espermatogênese. $\mathrm{Na}$ segunda etapa, a ação se faria sobre as células de Leydig, com diminuição dos níveis de testosterona. Esta diminuição acarretaria na parada da maturação espermática, na alteração da integridade funcional e estrutural do epidídimo e uma diminuição na concentração de testosterona plasmática (SCHEFFER et al., 2003).

A função testicular mais afetada pela varicocele é a espermatogênese. Os achados mais comuns na análise do sêmen são o aumento do número de formas patológicas 
de espermatozóides, diminuição da motilidade e diminuição da densidade de espermatozóides (PADUCH; SKOOG, 2001).

Hipóteses sobre os efeitos nocivos da varicocele incluem fatores do escroto, hipertermia testicular, elevações nos níveis séricos de gonadotrofinas, aumento da pressão venosa, acúmulo de toxinas, hipóxia e desequilíbrio hormonal. A varicocele também afeta os níveis de FSH e inibina B e são amplamente aceitos como marcadores do estado espermatogênico de um paciente. No entanto, há controvérsia sobre a natureza e o significado dos níveis séricos de hormônios (e particularmente níveis de inibina B) após o reparo da varicocele (PRASIVORAVONG et al., 2014).

Alguns estudos sugerem a correção cirúrgica da varicocele, a fim de evitar quaisquer efeitos negativos sobre a função testicular (KANTARTZI et al., 2007). Apesar das excelentes taxas de sucesso com o tratamento cirúrgico da varicocele, muitos profissionais ainda questionam a validade da realização da varicocelectomia e indicam tratamento com reprodução assistida (PASQUALOTTO et al., 2007).

\section{DIAGNÓSTICO}

A varicocele pode ser diagnosticada através de diversos meios, com ultrassonografia com Doppler, venografia, termografia escrotal, cintilografia e o exame físico que é a forma mais utilizado para o diagnóstico (WILL et al., 2011). Em comparação à venografia, a ultrassonografia com Doppler colorido tem sensibilidade e especificidade superiores a 90\%, enquanto a termografia escrotal e a cintilografia apresentam resultados variáveis (SADI et al., 2008).

O exame físico é fundamental para o diagnóstico da varicocele e deve ser realizado primeiro com o paciente em pé e depois deitado, em ambiente tranquilo e temperatura não refrigerada, o que favorece o relaxamento da musculatura escrotal (NARDOZZA, 2010). Este tem sido o método mais utilizado, apesar de possuir sensibilidade e especificidade de somente 70\% (PADUCH; SKOOG, 2001).

As varicoceles diagnosticadas pelo exame físico são classificadas de acordo com o tamanho. Varicoceles grau III são aquelas visíveis através da pele do escroto; as varicoceles grau II são aquelas palpadas sem o auxílio da manobra de Valsalva; enquanto as varicoceles grau I são aquelas palpadas somente com auxílio da manobra de Valsalva. Os outros exames não são capazes de diferenciar varicoceles clínicas e subclínicas (SADI et al., 2008).

\subsection{PREVALÊNCIA}

A incidência de varicocele varia com a idade, sendo de 7,2\% em indivíduos entre 2 e 19 anos, com a seguinte distribuição: 2 a 6 anos - 0,79\%; 7 a 10 anos 0,96\%; 11 a 14 anos - 7,8\% e 15 a 19 anos - 14,1\%. Após os 20 anos, a incidência é de $10 \%$ a $25 \%$. Em pacientes idosos, a varicocele está presente em até 42,9\%. Nessa faixa etária não existem estudos adequados que demonstrem existir relação entre varicocele e alterações hormonais (SADI et al., 2008).

Na população geral de homens saudáveis, a incidência geral de varicocele é de 10\% a 15\% (PADUCH; SKOOG, 2001). De 41\% dos homens que apresentam infertilidade primária, 19\% são causadas pela varicocele. E de $81 \%$ dos que apresentam infertilidade secundária e terciária, 45\% são causadas pela varicocele (COCUZZA et al., 2008). Já a incidência em adolescentes varia de $12,4 \%$ a $17,8 \%$, com uma média de $14,7 \%$, semelhante à incidência em homens adultos (PADUCH; SKOOG, 2001).

\subsection{TRATAMENTO MEDICAMENTOSO}

Existem poucos trabalhos sobre o tratamento medicamentoso da varicocele. O uso de carnitina associado a anti-inflamatório não hormonal por seis meses nos pacientes com varicocele clínica não se mostra eficaz. Na varicocele subclínica, o citrato de clomifeno não melhora a concentração e a motilidade espermática de maneira tão eficaz quanto a cirurgia, apesar de ambos não promoverem melhora seminal ou taxa de gravidez significativa. A associação de menotropina por três meses e varicocelectomia é benéfica quando comparada com o grupo tratado somente com cirurgia. Quanto mais precoce a introdução do tratamento clínico simultaneamente à cirurgia, melhor (SADI et al., 2008). 
O tratamento medicamentoso é para tentar melhorar a produção espermática e/ou a fertilidade de homens afetados. Os resultados destes estudos são muito controversos e, por isso, atualmente, o tratamento clínico não encontra respaldo de eficácia (SADI et al.,2008).

\subsection{TRATAMENTO CIRÚRGICO}

A varicocele é a causa tratável mais comum de infertilidade masculina. Pode ser corrigida através do tratamento cirúrgico (varicocelectomia) ou por embolização percutânea (GROBER et al., 2004). A reprodução assistida também demonstrou taxas de sucesso em relação a concepção, com o emprego de gametas obtidos diretamente dos testículos (NARDOZZA; REIS; CAMPOS, 2010).

A cirurgia pode ser realizada por meio de diversos métodos, mas a maioria dos especialistas em infertilidade realiza o tratamento por via inguinal ou subinguinal (PASQUALOTTO, 2007).

A via inguinal permite a ligadura das veias ao nível do anel inguinal interno e de vasos cremastéricos, porém necessita da abertura de fáscias, músculo e aponeurose. $\mathrm{Na}$ via subinguinal o procedimento é realizado com uma pequena incisão logo abaixo no anel inguinal externo. A musculatura e a fáscia local não são incisadas. Permite a identificação e ligadura de vasos cremastéricos responsáveis pela drenagem venosa do plexo pampiniforme. Como é um procedimento superficial a recuperação é mais rápida com menos dor no pós-operatório, pode ser feita com anestesia local, além de obter os melhores resultados com menos complicações (NARDOZZA; REIS; CAMPOS, 2010).

Tais técnicas aumentam a preservação das artérias e vasos linfáticos, diminuindo, desta forma, a probabilidade da recorrência ou persistência da varicocele.

A cirurgia laparoscópica tem sido utilizada para tratamento da varicocele; entretanto, esta técnica apresenta grande risco de complicações intraperitoneais como perfuração vesical, intestinal ou de grandes vasos sanguíneos (PASQUALOTTO, 2007).

A embolização percutânea para o reparo da varicocele pode estar associada com menos dor comparada à abordagem cirúrgica inguinal padrão, mas a disponibilidade de técnicos experientes em radiologia intervencionista limita a utilização desta técnica. Todas as abordagens cirúrgicas estão associadas com baixo risco de infecção da ferida operatória, hidrocele, recorrência ou persistência da varicocele, sangramento e raramente atrofia testicular (PASQUALOTTO, 2007).

O reparo da varicocele é claramente associado a uma significativa melhora na concentração de espermatozóides, bem como total a motilidade progressiva. $\mathrm{O}$ reparo da varicocele também contribui para reduzir o estresse oxidativo seminal e danos ao DNA do esperma. No entanto, existem evidências que demonstram um efeito benéfico de reparação da varicocele sobre as taxas de gravidez espontânea. Embora os vários métodos de reparação sejam todos viáveis, a reparação com a microcirurgia parece estar associada com melhores resultados e menores taxas de complicações (BAAZEEM et al., 2011).

Os efeitos da correção de varicocele sobre a qualidade do esperma e potencial reprodutivo são menos entendidos. A pesquisa clínica tem demonstrado uma melhora nos parâmetros seminais, integridade de DNA superior e uma melhor assistência aos resultados de tecnologia reprodutiva após a reparação de varicocele (McINTYRE; LIPSHULTZ, 2012).

Apesar das excelentes taxas de sucesso com o tratamento cirúrgico da varicocele, o interesse no tratamento cirúrgico ainda suscita controvérsias. Na literatura, a maioria dos estudos tem mostrado um aumento significativo na concentração e motilidade espermática após o tratamento cirúrgico, mas a melhora da morfologia dos espermatozóides parece não estar tão bem comprovada (JALLOULI et al., 2008). Muitos profissionais ainda questionam a validade da realização da varicocelectomia e indicam tratamento com reprodução assistida (PASQUALOTTO, 2007).

Homens de casais inférteis devem ser devidamente aconselhados sobre a probabilidade de alcançar uma melhoria significativa em parâmetros seminais após o reparo da varicocele. Resultados divergentes foram obtidos quando o critério de eficácia é uma melhora na morfologia espermática. O efeito de preparação de varicocele na cabeça do espermatozóide e parâmetros dos flagelos são menos claros. Alguns 
pesquisadores relataram aumento significativo na percentagem de espermatozóides com uma morfologia normal (NABI et al., 2004; GALFANO et al., 2009). Em contraste, Gazzera et al. não observaram diferenças significativas na morfologia do esperma.

Os resultados do estudo de Prasivoravong et al. mostram que a embolização de varicocele (uma técnica bem tolerada, com uma alta relação custo-benefício), em homens com varicocele grau III à esquerda, parâmetros anormais de esperma e infertilidade documentados, está associada com uma melhoria significativa na concentração de espermatozóides, motilidade, morfologia e vitalidade, mas não em níveis séricos de testosterona no paciente tratado, mas os níveis de FSH e inibina B não são afetados. Esta descoberta apoia sugestões que varicocele prejudica a espermatogênese, mas não a função de liberação de esteróides dos testículos.

\section{REPRODUÇÃO ASSISTIDA}

A área da reprodução assistida é um campo do conhecimento pertinente para pensar mudanças e progressos em que os avanços da ciência e da tecnologia oferecem inúmeros recursos para o tratamento da infertilidade humana (MOURA, 2009).

Nos últimos anos, houve um aumento considerável no número de casais inférteis que procuram as clínicas de reprodução assistida. Para muitos deles, essas técnicas de reprodução assistida são a última oportunidade para concretizar o sonho do filho biológico. Geralmente, elas são procuradas após um longo período de tentativas por meio de outros recursos (SANTOS, 2010).

Areprodução assistida implica em um conjunto de técnicas como relação programada, inseminação artificial intrauterina e fertilização extracorpórea que abrange a fertilização in vitro clássica e a fertilização in vitro por meio de injeção intracitoplasmática de espermatozóide (ICSI). Uma equipe multidisciplinar tem participação estreita no acompanhamento do desenvolvimento folicular, deteç̧ão e indução da postura ovular ou até mesmo a realização do encontro dos gametas, assim como na otimização da fase lútea
(FREITAS; SIQUEIRA; SAGRE, 2008).

Sawa et al. mostraram que as taxas de fertilização ICSI baixas também têm sido encontradas em homens com espermatozóides severamente alongados em comparação para outras anomalias de morfologia.

Com o advento das técnicas de reprodução assistida, muitos profissionais que tratam o casal infértil têm oferecido como tratamento de escolha a ICSI. Entretanto, estudos demonstram que a FIV/ICSI, além de não ser mais eficaz que a varicocelectomia, do ponto de vista econômico, ela é muito mais cara que o reparo cirúrgico da varicocele (PASQUALOTTO, 2007). Essa técnica envolve a seleção e a inserção de um único espermatozóide diretamente no citoplasma do ovócito, que ultrapassa todas as barreiras da fecundação, incluindo o complexo corona radiata, a zona pelúcida e o oolema, o que faz da ICSI uma técnica de micromanipulação mais invasiva. Sua maior vantagem é o fato da fertilização não ser afetada pela concentração, motilidade e/ou morfologia espermática. Sendo assim, a ICSI é hoje a técnica mais empregada nos casos em que existem alterações graves nos parâmetros seminais, como na varicocele (SANTOS, 2010).

Um estudo recente demonstrou que, em pacientes submetidos a um procedimento de reprodução assistida, após o procedimento cirúrgico, a porcentagem de mulheres que estabeleceu gravidez foi superior nos pacientes operados de varicocele (33,3\%), comparada a pacientes portadores de varicocele e que não foram submetidos ao procedimento cirúrgico de correção da varicocele (11\%) (PASQUALOTTO, 2007).

Sendo assim, o tratamento cirúrgico elimina com sucesso mais de $90 \%$ das varicoceles. A Associação Americana de Urologia e a Sociedade Americana de Medicina Reprodutiva recentemente descreveram que o reparo da varicocele deve ser considerado como a primeira opção de tratamento quando há presença de infertilidade associada com varicocele. Desta forma, pacientes com varicocele clinicamente detectável e esposas de idade inferior a 37 anos devem ser submetidos à varicocelectomia. Entretanto, quando a esposa tiver mais de 37 anos de idade, técnicas de reprodução assistida devem ser consideradas de primeira escolha (PASQUALOTTO, 2007). 


\section{CONCLUSÃO}

Avaricoceleéo achado clínico mais comum entre os homens inférteis. O diagnóstico definitivo baseia-se no baixo crescimento testicular e/ou veias salientadas no mesmo. A detecção da doença e/ou tratamento em tempo hábil elimina com sucesso mais de 90\% das varicoceles. A cirurgia de correção da varicocele (microcirúrgica subinguinal) é indicada em pacientes com varicocele clinicamente detectável, independente da histologia testicular, tipo de alteração na qualidade seminal ou níveis de FSH antes da cirurgia. Pacientes azoospérmicos talvez devam ser submetidos à varicocelectomia antes de serem encaminhados para clínicas de reprodução assistida. Quanto maior o grau de varicocele, maior é a chance de melhora na qualidade seminal após a varicocelectomia. Entretanto, pacientes com esposas com idade superior a 37 anos devem ser submetidos a técnicas de reprodução assistida e não varicocelectomia. No entanto os riscos cirúrgicos colocam em dúvida a realização do sonho, a paternidade condicionada à reprodução assistida ou os riscos cirúrgicos a serem enfrentados mesmo sendo a melhor opção. Estudos mais detalhados devem ser realizados e antes de conclusões confiáveis sobre o prognóstico para a fertilidade e o sucesso no tratamento e na obtenção de uma gravidez desejada.

\section{REFERÊNCIAS}

ABDELMASSIH, R Guia da Fertilidade. São Paulo: Tatiana Pronin, 2004.

BAAZEEM, A. et al. Varicocele and male factor infertility treatment: a new meta- analysis and review of the role of varicocele repair. Eur Urol., v. 60, n. 4, p. 796-808, 2011.

COCUZZA, M. et al. O papel da reparação varicocele na nova era da tecnologia de reprodução assistida. Clinics v. 63, n. 3. São Paulo, 2008.

COCUZZA, M. D. et al. Impact of clinical varicocele and testis size on seminal reactive oxygen species levels in a fertile population: a prospec tive controlled study. Fertil Steril., v. 90, p. 1103-1108, 2008.
COCUZZA, M. A. S. Avaliação do impacto da varicocele clinica no volume testicular, parâmetros seminais e níveis de radicais livres de oxigênio no sêmen de homens com infertilidade comprovada. 2011. 148f. Tese (Doutorado em Ciências) - Faculdade de Medicina da Universidade de São Paulo, SP.

CORRÊA, K. R. F. D. C. et al. Avaliação da eficácia adaptativa de mulheres e homens inseridos em um programa de fertilização in vitro. Psicol. Estud. v. 12, n. 2, 2007.

FREITAS, M.; SIQUEIRA, A. A. F.; SEGRE, C. A. M. Avanços em reprodução assistida. Rev. bras. Crescimento Desenvolv., v. 18, n. 1, 2008.

GALFANO, A.; NOVARA, G.; IAFRATE, M.; DE MARCO, V.; COSENTINO, M.; D'ELIA, C.; ARTIBANI, W.; FICARRA, V. Improvement of seminal parameters and pregnancy rates after antegrade sclerotherapy of internal spermatic veins. Fertil Steril., v. 91, p. 1085-1089, 2009.

GAZZERA, C.; RAMPADO, O.; SAVIO, L.; Di BISCEGLIE, C.; MANIERI, C.; GANDINI, G. Radiological treatment of male varicocele: technical, clinical, seminal and dosimetric aspects. Radiol Med (Torino), v. 111, p. 449-458, 2006.

GROBER, E. D. et al. Microsurgical treatment of persistent or recurrent varicocele. Fertility and sterility., v. 82, n. 3, 2004.

JALLOULI, $\mathrm{H}$. et al. Le traitement chirurgical de la varicocèle améliore la fertilité et aide à la procréation médicalement assistée. Progrès en urologie. v.18, p. 543-549, 2008

KANTARTZI, P. P. et al. A infertilidade masculina e varicocele: mitos e realidade. Rev. Hippokratia. v.11, n. $2,2007$.

LEWIS, R. Genética Humana: Conceitos e aplicações. $5^{\mathrm{a}}$ ed. Rio de Janeiro: Guanabara Koogan, 2004.

McINYRE, M.; HSIEH, T. C.; LIPSHULTZ, L. Varicocele repair in the era of modern assisted reproductive techniques. Curr Opin Urol, 22:517-520, 2012. 
MENKVELD, R.; HOLLEBOOM, C. A. G.; RHEMREV, J. P. T. Measurement and significance of sperm morphology. Asian J Androl 2011, 13:59-68.

MOURA, M. D. et al. Reprodução assistida. Um pouco de história. Rev. SBPH. v. 12, n. 2, 2009.

NABI, G.; ASTERLINGS, S.; GREENE, D. R.; MARSH, R. L. Percutaneous embolization of varicoceles: outcomes and correlation of semen improvement with pregnancy. Urology, v. 63, p. 359-363, 2004.

NARDOZZA, A. J.; REIS, R. B.; CAMPOS, R. S. M. MANU: manual de urologia. São Paulo: Planmark, 2010.

PADUCH, D. U.; SKOOG, S. J. Gestão atual de Varicocele Adolescente. Rev.Urol. v. 3, n. 3, p. 120-133, 2001.

PASQUALOTTO, F. F. Investigação e reprodução assistida no tratamento da infertilidade masculina. Rev. Bras. Ginecol. Obstet., v. 29, n. 2, 2007.

PASQUALOTTO, F. F.; OGLIARI, K. S.; PASQUALOTTO, E. B. Atualidades no tratamento do homem infértil com varicocele. Reproducción humana, 2007.

PRASIVORAVONG, J. et al. Beneficial effects of varicocele embolization on semen parameters. Basic and Clinical Andrology, v. 24, n. 9, p. 1-6, 2014.

SAADE, R D. Infertilidade masculina. Disponível em: $<$ http://www.fcm.unicamp.br/deptos/urologia/graduacao/pdf/ Infertilidade_Masculina.pdf> Acesso em: 18ago. 2012.

SADI, M. et al. Varicocele. [s.l.]: Associação Médica Brasileira e Conselho Federal de Medicina, 2008.

SANTOS, M. F. O. Injeção intracitoplasmática de espermatozóides: questões éticas e legais. Rev. Bras. Saude Mater. Infant. v. 10, n. 2, 2010.

SCHEFFER, B. B. et al. Reprodução humana assistida. São Paulo: Atheneu, 2003.

SOLIGO, A. G. S. et al. Prevalência dos fatores trombofílicos em mulheres com infertilidade. Rev. Bras.

Ginecol. Obstet. v. 29, n. 5. Rio de Janeiro, 2007.
VASQUEZ, D. V. et al. Testicular varicocele em adolescentes. Rev. Saúde Uninor. v. 25, n. 2, 2009.

WILL, M. A. et al. The great debate: varicocele treatment and impact on fertility. Fertil Steril, n. 95, p. 841-852, 2011.

Recebido em: 11 de agosto de 2013 Aceito em: 03 de março de 2015 